\title{
Ophthalmic Insert Dosage Form
}

National Cancer Institute

\section{Source}

National Cancer Institute. Ophthalmic Insert Dosage Form. NCI Thesaurus. Code C149704.

Solid sterile preparation of suitable size and shape, designed to be inserted in the conjunctival sac to produce a local or ocular effect by the release of active substance(s) over a determined period of time. Ophthalmic inserts generally consist of a reservoir of active substance(s) embedded in a matrix or bounded by a rate-controlling membrane. They are presented individually in sterile containers. 